\title{
Trans-arterial radioembolization in intermediate-advanced hepatocellular carcinoma: systematic review and meta-analyses
}

\author{
Carla Rognoni ${ }^{1}$, Oriana Ciani ${ }^{1,2}$, Silvia Sommariva ${ }^{1}$, Antonio Facciorusso ${ }^{3}$, Rosanna \\ Tarricone $^{1,4}$, Sherrie Bhoori ${ }^{3}$ and Vincenzo Mazzaferro ${ }^{3}$ \\ ${ }^{1}$ Centre for Research on Health and Social Care Management (CERGAS), Bocconi University, Milan, Italy \\ ${ }^{2}$ Evidence Synthesis and Modelling for Health Improvement (ESMI), University of Exeter Medical School, South Cloisters, St \\ Luke's Campus, Exeter, UK \\ ${ }^{3}$ Department of Surgery, Liver Surgery, Transplantation and Gastroenterology, Istituto Nazionale Tumori Fondazione IRCCS, \\ National Cancer Institute of Milan, and University of Milan, Milan, Italy \\ ${ }^{4}$ Department of Policy Analysis and Public Management, Bocconi University, Milan, Italy \\ Correspondence to: Carla Rognoni, email: carla.rognoni@unibocconi.it \\ Keywords: hepatocellular carcinoma, intermediate stage, advanced stage, trans-arterial radioembolization, meta-analysis \\ Received: April 16, $2016 \quad$ Accepted: July 10, $2016 \quad$ Published: August 26, 2016
}

\section{ABSTRACT}

Trans-arterial radioembolization (TARE) is a recognized, although not explicitly recommended, experimental therapy for unresectable hepatocellular carcinoma (HCC).

A systematic literature review was performed to identify published studies on the use of TARE in intermediate and advanced stages HCC exploring the efficacy and safety of this innovative treatment.

Twenty-one studies reporting data on overall survival (OS) and time to progression (TTP), were included in a meta-analysis. The pooled post-TARE OS was $63 \%$ (95\% CI: $56-70 \%$ ) and $27 \%$ (95\% CI: $21-33 \%)$ at $1-$ and 3-years respectively in intermediate stage HCC, whereas OS was 37\% (95\% CI: $26-50 \%$ ) and $13 \%$ (95\% CI: 9-18\%) at the same time intervals in patients with sufficient liver function (Child-Pugh A-B7) but with an advanced HCC because of the presence of portal vein thrombosis. When an intermediate and advanced case-mix was considered, OS was $58 \%$ (95\% CI: $48-67 \%$ ) and 17\% (95\% CI: $12-23 \%)$ at 1- and 3-years respectively. As for TTP, only four studies reported data: the observed progression probability was $56 \%$ (95\% CI: $41-70 \%)$ and 73\% (95\% CI: 56-87\%) at 1 and 2 years respectively. The safety analysis, focused on the risk of liver decompensation after TARE, revealed a great variability, from $0-1 \%$ to more than $36 \%$ events, influenced by the number of procedures, patient Child-Pugh stage and treatment duration.

Evidence supporting the use of radioembolization in HCC is mainly based on retrospective and prospective cohort studies. Based on this evidence, until the results of the ongoing randomized trials become available, radioembolization appears to be a viable treatment option for intermediate-advanced stage HCC.

\section{INTRODUCTION}

Hepatocellular carcinoma represents the third most common cause of cancer death, causing nearly 746,000 deaths per year in the world $[1,2]$. In almost all cases, one risk factor can be identified alone or in combination with others, with cirrhosis being the most frequent of them [3]. Prognosis of patients with $\mathrm{HCC}$ is poor, with a ratio of mortality to incidence of 0.95 [2]. Indeed HCC represents a major global health problem, also considering that its incidence increases progressively with aging of population [4].

The Child-Pugh score is used to assess the prognosis of chronic liver disease, mainly cirrhosis [5]. Patients are classified according to different expected survivals from A to C (two year survival: A $85 \%$, B 57\%, C 35\%) based on five clinical measures of liver disease (total bilirubin, serum albumin, prothrombin time, ascites, hepatic encephalopathy). The American and European Societies for the Study of the Liver endorse the Barcelona Clinic 
Liver Cancer (BCLC) classification system for staging and allocation to treatment in patients with HCC [6]. Using this classification, as others applied in the Asia-Pacific Countries, the therapeutic algorithm varies depending on the stage of disease, ranging from curative treatments (such as resection, ablation or transplantation) to palliation and best supportive care. Overall, only a minority of patients with $\mathrm{HCC}$ receive curative treatments and no more than 5\% are eligible for liver transplantation [7].

In current practice, advanced stage patients present as such in $60-70 \%$ of cases while the rest progresses to this stage despite treatment of more precocious cancer presentations [6]. Prospective randomized controlled trials (RCTs) have shown that sorafenib prolongs survival in all subtypes of advanced HCC patients $[8,9]$ but side effects may lead to discontinuation of this treatment in up to $45 \%$ of patients. As a consequence, a significant number of advanced HCC patients may be precluded from the therapeutic benefit of sorafenib for related toxicities $[10$, $11]$.

In the intermediate HCC stage, a wide range of interventional loco-regional treatments are available and trans-arterial chemoembolization (TACE) variously administered, is considered as the standard of care [4, 6]. Also in advanced HCC - particularly when tumour extension is confined to the liver and when it is associated with a preserved hepatic function - the search for a tolerated loco-regional intervention able to challenge systemic therapy has been repeatedly investigated.

Trans-arterial radioembolization (TARE), often referred as a form of selective internal radiation therapy (SIRT) is a recognized, although not explicitly recommended therapy in several guidelines on clinical management of non-resectable HCC [4, 6, 12]. According to the latest release of the ESMO guidelines, however, TARE may compete with sorafenib in intermediate stage patients with prior TACE failure or advanced patients with tumoural macrovascular invasion (i.e. portal vein thrombosis, PVT) with no extra-hepatic spread and good liver function [12].

On technical grounds, TARE is a catheter-based interventional procedure that allows the emission of $\beta$-radiations at therapeutic levels directly into the tumour through its feeding arteries. Such a delivery mechanism, as for TACE, is aimed at minimizing the damage to the healthy liver parenchyma adjacent to the tumour. The main mechanism of action of TARE is a local brachytherapy and, unlike TACE, it doesn't result in microvascular embolization and tumour ischemia [13]. Devices for radioembolization are commercially available in both form of implantable glass (Therasphere ${ }^{\circledR}$ ) or biocompatible resin-based (SIR-Spheres ${ }^{\circledR}$ ) radioactive $\left(\mathrm{Yttrium}^{90}-\mathrm{Y}^{90}\right.$ ) spheres [13].

In the light of the increasing use of this innovative therapy in clinical practice, this study aims to systematically review the available evidence for the clinical efficacy and safety of TARE (glass or resin-based) in patients with intermediate-advanced stages HCC.

\section{RESULTS}

Out of 6641 references screened, 26 studies met the selection criteria. The study selection process is summarized in Figure 1.

The vast majority of included studies were observational cohorts, however three comparative studies were found: two RCTs comparing TARE with either TARE + sorafenib [14] or TACE [15] and a propensityscore matching design study with sorafenib [16]. In Supplementary Table 1 a detailed description of all 26 studies is given.

A summary of the critical appraisal of TARE studies is reported in Supplementary Table 2.

All studies showed low to medium methodological quality. One study [20] did not present clearly inclusion and exclusion criteria and only 3 studies $[15,27,36]$ were conducted in more than one centre. No therapy was reported as concurrently administered but in 7 studies follow-on treatments were described. Nine studies had a prospective design while dropouts were adequately described in only 3 studies.

\section{Outcome measures}

Studies presenting OS or TTP data, stratified by presence or absence of PVT were all included in the metaanalyses except for two studies, one which did not report survival curves [17] and the second [37] which reported only survival data stratified by treatment dose, good or poor candidate groups and surgery vs. no surgery groups.

Studies considering patients with extrahepatic spread [26] or patients with deteriorated liver function (Child-Pugh C stage) [18] or patients awaiting liver transplantation [14] were excluded from the analysis.

Details on the 21 studies included in the quantitative analyses are listed in Table 1 together with patients and treatment characteristics.

The pooled OS was $63 \%$ (95\% CI: $56-70 \%)$ and $27 \%$ (95\% CI: $21-33 \%)$ at 1- and 3-years respectively, for the population receiving TARE at an intermediateadvanced stage (namely in unresectable HCC lacking demonstration of PVT: Figure 2A), whereas OS was $37 \%$ (95\% CI: $26-50 \%)$ and $13 \%$ (95\% CI: 9-18\%) at the same follow-up times in patients with sufficient liver function but with an advanced HCC because of presence of PVT (Figure 2B).

When the entire population of patients receiving TARE was considered, with no stratification on PVT i.e. with a case-mix of intermediate and advanced HCC - the OS was $58 \%$ (95\% CI: $48-67 \%)$ and $17 \%$ (95\% CI: $12-23 \%$ ) at 1 - and 3-years respectively (Supplementary 
Table 1: Characteristics of studies on radioembolization (TARE) included in the meta-analysis.

\begin{tabular}{|c|c|c|c|c|c|}
\hline Study & Study type & Patients characteristics & Child-Pugh (A-B-C) & $\begin{array}{l}\text { \% Patients } \\
\text { with PVT }\end{array}$ & $\begin{array}{l}\text { Narm } \\
\text { TARE }\end{array}$ \\
\hline $\begin{array}{l}\text { Woodall } 2009 \\
{[19]}\end{array}$ & Cohort & $\begin{array}{l}20 \text { patients without PVT who } \\
\text { received SIR, } 15 \text { patients with } \\
\text { PVT who were treated. Of the PVT } \\
\text { patients treated, } 67 \% \text { had portal VT, } \\
7 \% \text { had cava PVT, and } 26 \% \text { had both }\end{array}$ & 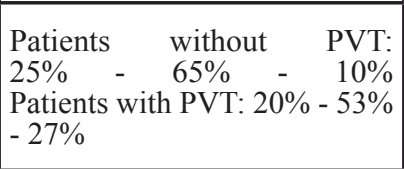 & $43 \%$ & 35 \\
\hline Carr 2010 [20] & Cohort & \begin{tabular}{|l|} 
HCC (with or without PVT) \\
considered unsuitable for resection, \\
RFA, or liver transplantation
\end{tabular} & NA & $28 \%$ & 99 \\
\hline Hilgard 2010 [21] & Cohort & $\begin{array}{l}\text { Advanced HCC and liver cirrhosis } \\
\text { (with or without PVT) }\end{array}$ & $78 \%-22 \%-0 \%$ & $31 \%$ & 108 \\
\hline Kooby 2010 [22] & Cohort & $\begin{array}{l}\text { Unresectable HCC (with or without } \\
\text { PVT) }\end{array}$ & $48 \%-52 \%-0 \%$ & $52 \%$ & 27 \\
\hline Salem 2010 [23] & Cohort & HCC (with or without PVT) & $45 \%-52 \%-3 \%$ & $43 \%$ & 291 \\
\hline Tsai 2010 [24] & Cohort & $\begin{array}{l}\text { HCC with main }(n=10) \text { or first }(n=12) \\
\text { branch PVT }\end{array}$ & $55 \%-27 \%-5 \%(N A 13 \%)$ & $100 \%$ & 22 \\
\hline $\begin{array}{l}\text { Lambert } 2011 \\
{[25]}\end{array}$ & Cohort & $\begin{array}{l}\text { Patients suffering from HCC (with } \\
\text { or without PVT) with the disease } \\
\text { confined to the liver but not amenable } \\
\text { to surgery, RFA or transplantation. }\end{array}$ & $92 \%-8 \%-0 \%$ & $7 \%$ & 29 \\
\hline Sangro 2011 [27] & $\begin{array}{l}\text { Cohort } \\
\text { (retrospective) }\end{array}$ & $\begin{array}{l}\text { Patients with unresectable HCC (with } \\
\text { or without PVT) }\end{array}$ & $83 \%-17 \%-0 \%$ & $23 \%$ & 325 \\
\hline $\begin{array}{l}\text { Mazzaferro } 2013 \\
{[28]}\end{array}$ & Phase II & $\begin{array}{l}\text { Consecutive cohort of } 52 \text { patients } \\
\text { with liver cirrhosis and HCC (with } \\
\text { or without PVT) confined to the } \\
\text { liver and not eligible to conventional } \\
\text { curative treatments }\end{array}$ & $83 \%-17 \%-0 \%$ & $67 \%$ & 52 \\
\hline $\begin{array}{l}\text { Moreno-Luna } \\
2013[29]\end{array}$ & $\begin{array}{l}\text { Cohort } \\
\text { (retrospective) }\end{array}$ & $\begin{array}{l}\text { patients with unresectable } \mathrm{HCC} \\
\text { without PVT }\end{array}$ & $87 \% \quad-13 \% \quad-\quad 0 \%$ & $0 \%$ & 61 \\
\hline Weng 2013 [30] & Cohort & HCC patients with PVT & NA & $100 \%$ & 149 \\
\hline $\begin{array}{l}\text { Gramenzi } 2014 \\
{[16]}\end{array}$ & $\begin{array}{l}\text { Cohort } \\
\text { (retrospective) }\end{array}$ & HCC patients (with or without PVT) & $91 \% \quad-\quad 9 \% \quad-\quad 0 \%$ & $41 \%$ & 32 \\
\hline Khor 2014 [31] & Cohort & $\begin{array}{l}\text { Patients with HCC (with or without } \\
\text { PVT) unsuitable for surgical resection }\end{array}$ & $59 \%-38 \%-3 \%$ & $31 \%$ & 103 \\
\hline Kwok 2014 [32] & Cohort & $\begin{array}{l}\text { Inoperable HCC (with or without } \\
\text { PVT) }\end{array}$ & $83 \%-17 \%-0 \%$ & $63 \%$ & 30 \\
\hline Padia 2014 [33] & Cohort & $\begin{array}{l}\text { Unresectable HCC (with or without } \\
\text { PVT) }\end{array}$ & $55 \%-40 \%-5 \%$ & $60 \%$ & 20 \\
\hline Saxena 2014 [34] & $\begin{array}{l}\text { Cohort } \\
\text { (retrospective) }\end{array}$ & $\begin{array}{l}\text { HCC patients not amenable to } \\
\text { curative surgical resection }\end{array}$ & $67 \%-22 \%-2 \%(N A 9 \%)$ & NA & 45 \\
\hline She 2014 [35] & Cohort & $\begin{array}{l}\text { Patients with unresectable advanced } \\
\text { HCC (with or without PVT) }\end{array}$ & $94 \%-6 \%-0 \%$ & $50 \%$ & 16 \\
\hline $\begin{array}{l}\text { El Fouly } 2015 \\
{[36]}\end{array}$ & Cohort & $\begin{array}{l}\text { HCCs classified as intermediate stage } \\
\text { (BCLC B) without PVT }\end{array}$ & $84 \%-16 \%-0 \%$ & $0 \%$ & 44 \\
\hline $\begin{array}{l}\text { Kolligs } \quad 2015 \\
{[15]}\end{array}$ & $\mathrm{RCT}$ & $\begin{array}{l}\text { Patients with unresectable HCC, } \\
\text { Child-Pugh } \leq \text { B7, ECOG performance } \\
\text { status } \leq 2 \text { and } \leq 5 \text { liver lesions without } \\
\text { extrahepatic spread, without PVT }\end{array}$ & $92 \%-8 \%-0 \%$ & $0 \%$ & 13 \\
\hline Ozkan 2015 [38] & Cohort & HCC patients (with or without PVT) & $90 \%-10 \%-0 \%$ & $41 \%$ & 29 \\
\hline Soydal 2015 [39] & $\begin{array}{l}\text { Cohort } \\
\text { (retrospective) }\end{array}$ & $\begin{array}{l}\text { Patients with unresectable } \mathrm{HCC} \\
\text { lesions }\end{array}$ & NA & NA & 28 \\
\hline
\end{tabular}

$\mathrm{HCC}=$ Hepatocellular carcinoma, PVT $=$ portal vein thrombosis, TARE $=$ trans-arterial embolization, $\mathrm{BCLC}=\mathrm{Barcelona}$ Clinic Liver Cancer classification, $\mathrm{ECOG}=$ Eastern Cooperative Oncology Group, NA = not available 
Figure 1). In the cumulative analysis the percentage of patients with PVT was 38\% while those with suboptimal liver function (Child-Pugh non-A) were 21\%. Both these well-known prognostic variables likely influenced the observed results, although a direct evidence of that could not be inferred from the available data.

In terms of TTP, only four studies reported clear data: two of them had non-stratified TTP curves [21, 36], in one study data were stratified by Child-Pugh stage and BCLC classifications [23] and only one study stratified data on presence/absence of PVT [28]. Cumulative probability of progression in intermediate-advanced HCC lacking PVT at 1- and 2-years was 41\% (95\% CI: 29-54\%) and 64\% (95\% CI: 52-76\%) respectively (Figure 3A) while in advanced HCC carrying PVT TTP increased to $69 \%$ (95\% CI: $51-83 \%$ ) at both 1 and 2 years in a single study (Figure 3B). When the entire population of patients receiving TARE - with intermediate $55 \%$ and advanced $45 \%$ HCC - were pooled, the observed progression probability was 56\% (95\% CI: $41-70 \%)$ and $73 \%(95 \%$ CI: $56-87 \%$ ) at 1 and 2 years respectively (Supplementary Figure 2).

All results were confirmed in fixed effect metaanalysis models (data not shown).

\section{Liver impairment after TARE}

As described above, tumour progression after TARE was reported as a late event mainly due to deterioration of performance status and of Child-Pugh stage.

More specifically, the analysis on the adverse events related to TARE was focused on liver decompensation and/or failure following treatment. There was a large heterogeneity in definition of liver decompensation in the included studies and with that bias the percentage of patients suffering liver decompensation after TARE is reported in Table 2. In particular, TARE-related liver impairment events ranged from $0-1 \%$ in some studies to $36.5 \%$ in others depending on the number of treatments received, performance status, duration and type of preTARE treatments. Liver decompensation was more likely in patients with multiple pre-TARE therapies and borderline cirrhosis (HR: 3.2; 95\% CI: 1.8-9.7) as previously reported [40]. Medium time from TARE to liver decompensation was 3 months (range: 1-6).

\section{DISCUSSION}

In the last decade trans-arterial radioembolization has emerged as a viable loco-regional treatment option for patients with unresectable hepatocellular carcinoma associated or not with portal vein thrombosis, although its use is still not formally recommended in clinical guidelines due to lack of prospective randomized studies. While large RCTs have been implemented and likely to be reported in the near future, the present study aimed at identifying the available evidence around the efficacy and safety of TARE through a systematic review of published

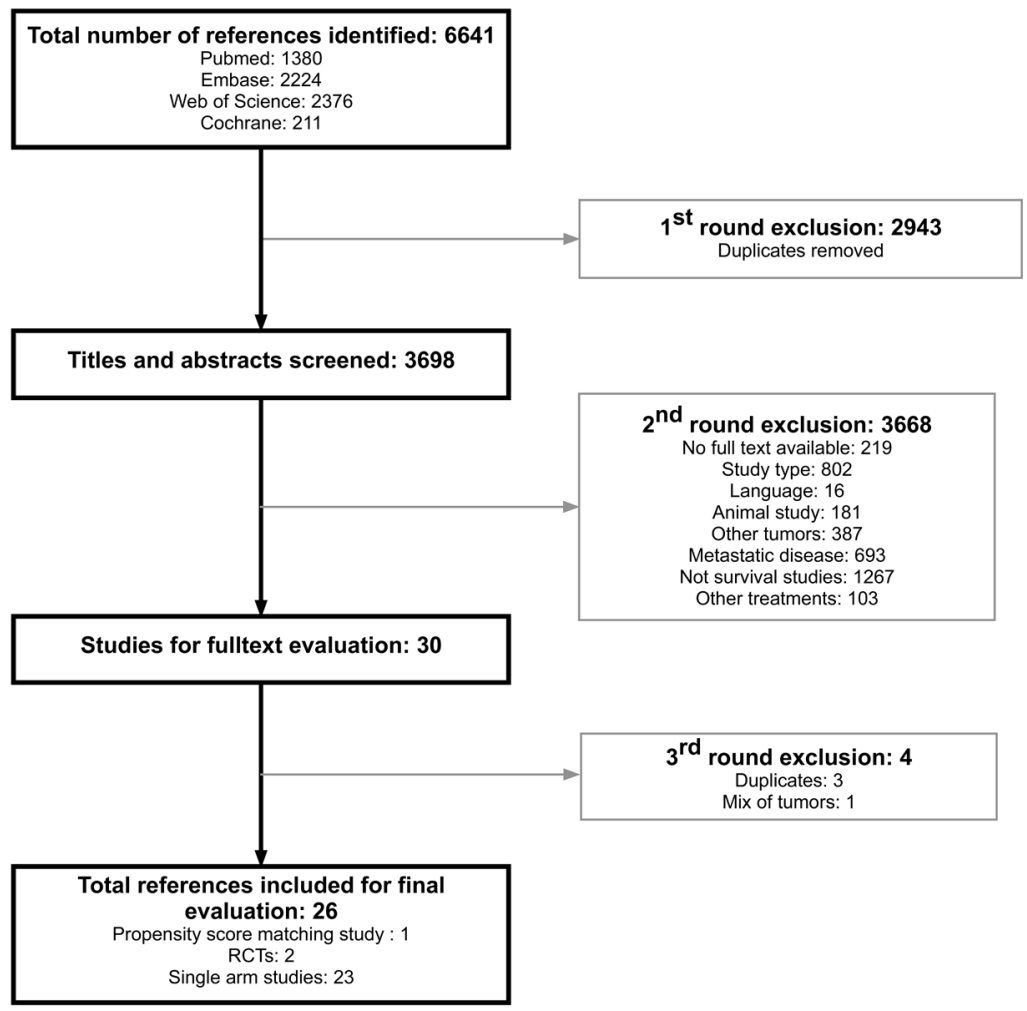

Figure 1: Study flow chart. 
Table 2: Liver failure after TARE and study characteristics.

\begin{tabular}{|c|c|c|c|c|c|c|}
\hline Study & $\begin{array}{l}\% \text { of patients } \\
\text { with liver } \\
\text { impairment } \\
\end{array}$ & \begin{tabular}{|l|}
$\begin{array}{l}\text { Time from } \\
\text { TARE } \\
\text { (months) }\end{array}$ \\
\end{tabular} & \begin{tabular}{|l|} 
Mean number of \\
treatments per \\
patient
\end{tabular} & Intervention & Mean dose & Previous treatments \\
\hline $\begin{array}{l}\text { Woodall } \\
2009 \text { [19] }\end{array}$ & $10 \%$ & $\mathrm{NC}$ & $\begin{array}{l}\text { PVT patients: } 2 ; \text { no } \\
\text { PVT patients: } 1.5 \\
\end{array}$ & TARE (Therasphere) & $\begin{array}{l}\text { median dose of } 120 \\
\text { Gy (range 120-142) } \\
\end{array}$ & NA \\
\hline $\begin{array}{l}\text { Carr } 2010 \\
{[20]}\end{array}$ & NA & NA & $\begin{array}{l}\text { Single, planned } \\
\text { treatment, but } 30 \% \\
\text { required a second } \\
\text { treatment because of } \\
\text { new, late appearing } \\
\text { lesions } \\
\end{array}$ & TARE (Therasphere) & $\begin{array}{l}\text { from } 135 \text { to } 150 \mathrm{~Gy} \\
\text { to the treated lobe }\end{array}$ & NA \\
\hline $\begin{array}{l}\text { Hilgard } 2010 \\
{[21]}\end{array}$ & $2.78 \%$ & 1 & 1.47 & TARE (Therasphere) & $120 \pm 8 \mathrm{~Gy}$ & $\begin{array}{l}62 \% \text { of patients were } \\
\text { therapy-naive; the rest } \\
\text { received prior local } \\
\text { therapy with curative } \\
\text { or palliative intent }\end{array}$ \\
\hline $\begin{array}{l}\text { Kooby } 2010 \\
{[22]}\end{array}$ & $22 \%$ & 1 & $1.2 \pm 1.1$ & TARE (SIR-Spheres) & $\begin{array}{l}740-2220 \mathrm{MBq} \text { per } \\
\text { lobe }\end{array}$ & NA \\
\hline $\begin{array}{l}\text { Salem } 2010 \\
\text { [23] }\end{array}$ & $19 \%$ & $\mathrm{NC}$ & 1.8 & TARE (Therasphere) & $\begin{array}{l}\text { The median dose } \\
\text { was } 103 \text { Gy per } \\
\text { treatment }(95 \% \text { CI } \\
99-108)\end{array}$ & $\begin{array}{l}\text { Resection } 5 \%, \text { RFA } \\
2 \% \text {, TACE 5\%, } \\
\text { Orthotopic liver } \\
\text { transplantation } 1 \% \\
\end{array}$ \\
\hline $\begin{array}{l}\text { Tsai } 2010 \\
{[24]}\end{array}$ & $13 \% *$ & 1 & 1.45 & $\begin{array}{l}\text { Resin or glass } \\
\text { microspheres }\end{array}$ & $\begin{array}{l}\text { 2.7 GBq (range } \\
0.59-9.21)\end{array}$ & NA \\
\hline $\begin{array}{l}\text { Lambert } \\
2011[25]\end{array}$ & $3 \%$ & $\begin{array}{l}\text { in the } \\
\text { weeks } \\
\text { following } \\
\text { treatment }\end{array}$ & 1.2 & TARE (Therasphere) & $\begin{array}{l}2.17 \mathrm{GBq} \text {, range } \\
0.73 \text { to } 3.99 \mathrm{GBq}\end{array}$ & $\begin{array}{l}\text { RFA } 13.7 \% \text {, Liver } \\
\text { resection and } \\
\text { radiolabelled Lipiodol } \\
3.4 \% \text {, Transplantation } \\
\text { and subsequent } \\
\text { sorafenib 3.4\% } \\
\end{array}$ \\
\hline $\begin{array}{l}\text { Sangro } 2011 \\
{[27]}\end{array}$ & $5.80 \%$ & 3 & 1.08 & TARE (Therasphere) & $\begin{array}{l}1.6 \mathrm{GBq} \text { (range } \\
0.3-4.0)\end{array}$ & $\begin{array}{l}\text { TARE or TACE } \\
(27.4 \%) \text {, surgical } \\
\text { resection or } \\
\text { transplantation } \\
(18.2 \%), \\
\text { percutaneous ablation } \\
(9.2 \%)\end{array}$ \\
\hline $\begin{array}{l}\text { Mazzaferro } \\
2013 \text { [28] }\end{array}$ & $36.50 \%$ & 6 & 1.12 & TARE (Therasphere) & $\begin{array}{l}\text { median 2.6 GBq } \\
\text { (range 1.1-5.7); } \\
\text { median dose to liver } \\
\text { lobe 101 Gy per } \\
\text { treatment (range } \\
\text { 34-146) }\end{array}$ & $\begin{array}{l}\text { RFA } 13.5 \% \text {, Liver } \\
\text { resection } 15.4 \%\end{array}$ \\
\hline $\begin{array}{l}\text { Moreno- } \\
\text { Luna 2013 } \\
{[29]}\end{array}$ & $0 \%$ & & 1.28 & TARE (Therasphere) & $\begin{array}{l}\text { The target dose of } \\
80-150 \text { Gy }\end{array}$ & NA \\
\hline $\begin{array}{l}\text { Weng } 2013 \\
{[30]}\end{array}$ & NA & & NA & TARE (Therasphere) & NA & NA \\
\hline $\begin{array}{l}\text { Gramenzi } \\
2014[16]\end{array}$ & $\begin{array}{l}9 \% \text { (grade } \\
3-4) \text { in the } \\
\text { whole group } \\
\text { of patients }\end{array}$ & 6 & $\begin{array}{l}\text { Median tumour dose } \\
263.2 \text { Gy (range 16.6- } \\
\text { 1145.8); Total injected } \\
\text { activity } 1.83 \text { GBq } \\
(0.45-2.41)\end{array}$ & TARE (SIR-Spheres) & $\begin{array}{l}\text { median tumor } \\
\text { dose was 119.8 Gy } \\
\text { (range, 31.4-420.2 } \\
\text { Gy) }\end{array}$ & $\begin{array}{l}\text { Resection } 13.6 \% \text {, } \\
\text { RFA } 12.6 \%\end{array}$ \\
\hline $\begin{array}{l}\text { Khor } 2014 \\
{[31]}\end{array}$ & $1 \%$ & $\mathrm{NC}$ & 1.175 & TARE (SIR-Spheres) & $\begin{array}{l}\text { All patients } \\
\text { received more than } \\
2 \mathrm{GBq} \text { of Y90 }\end{array}$ & NA \\
\hline $\begin{array}{l}\text { Kwok } 2014 \\
{[32]}\end{array}$ & $13 \%$ & 3 & NA & TARE (SIR-Spheres) & $\begin{array}{l}\text { Median dose to } \\
\text { treated segment } \\
\text { 254Gy, median } \\
\text { dose to the tumor } \\
536 \mathrm{~Gy}\end{array}$ & $\begin{array}{l}\text { Ablation } 20 \% \text {, } \\
\text { chemoembolization } \\
5 \% \text {, radioembolization } \\
5 \%\end{array}$ \\
\hline
\end{tabular}




\begin{tabular}{|c|c|c|c|c|c|c|}
\hline $\begin{array}{l}\text { Padia } 2014 \\
\text { [33] }\end{array}$ & $10 \%$ & $\mathrm{NC}$ & 1 & TARE (Therasphere) & $\begin{array}{l}1.49 \mathrm{GBq} \text { (range } \\
0.34-2.50)\end{array}$ & $\begin{array}{l}\text { Liver resection } \\
13 \% \text {, transarterial } \\
\text { chemoembolization } \\
\text { or hepatic artery } \\
\text { chemoinfusion } 24 \% \text {, } \\
\text { ablative therapy } \\
9 \% \text {, at least one } \\
\text { line of systemic } \\
\text { chemotherapy } 13 \%\end{array}$ \\
\hline $\begin{array}{l}\text { Saxena } 2014 \\
{[34]}\end{array}$ & $4.40 \%$ & $\mathrm{NC}$ & NA & $\begin{array}{l}\text { TARE (SIR- } \\
\text { Spheres) }\end{array}$ & NA & NA \\
\hline $\begin{array}{l}\text { She } 2014 \\
{[35]}\end{array}$ & $6.30 \%$ & $\mathrm{NC}$ & NA & $\begin{array}{l}\text { TARE (not } \\
\text { specified) }\end{array}$ & NA & $\begin{array}{l}\text { Resection } 7 \% \text {, RFA } \\
11 \% \text {, TACE } 32 \%\end{array}$ \\
\hline $\begin{array}{l}\text { El Fouly } \\
2015[36]\end{array}$ & $9.09 \%$ & 1 & $1.4 \pm 0.6$ & TARE (Therasphere) & $\begin{array}{l}\text { median } 1.6 \pm 0.6 \\
\text { GBq }\end{array}$ & NA \\
\hline $\begin{array}{l}\text { Kolligs } \\
2015[15] \\
\end{array}$ & $<5 \%$ & 4 & 1 & \begin{tabular}{|l} 
TARE (SIR- \\
Spheres) \\
\end{tabular} & \begin{tabular}{|l|}
$1.5 \mathrm{GBq}$ (range \\
$1-2.2)$ \\
\end{tabular} & $34.50 \%$ \\
\hline $\begin{array}{l}\text { Ozkan } 2015 \\
{[38]}\end{array}$ & $0 \%$ & & NA & $\begin{array}{l}\text { resin or glass } \\
\text { microspheres }\end{array}$ & $1.5 \pm 0.2 \mathrm{GBq}$ & $\begin{array}{l}\text { None of them had } \\
\text { received prior } \\
\text { treatment before SIRT }\end{array}$ \\
\hline $\begin{array}{l}\text { Soydal } 2015 \\
\text { [39] }\end{array}$ & NA & NA & $\begin{array}{l}\text { Treatment was applied } \\
\text { to the right lobe in } 22 \\
\text { patients and both lobes } \\
\text { in } 6 \text { patients }\end{array}$ & TARE (resin) & NA & NA \\
\hline
\end{tabular}

$* \%$ on the number of treatments performed

$\mathrm{TARE}=$ trans-arterial radioembolization, TACE $=$ trans-arterial chemoembolization, $\mathrm{RFA}=$ radio-frequency ablation, SIRT $=$ selective internal radiation therapy, $\mathrm{NC}=$ not clear, $\mathrm{NA}=$ not available

studies conducted on patients with intermediate and advanced HCC.

We identified 23 observational cohort studies, two RCTs of TARE vs. TARE + sorafenib and TARE vs. TACE and one propensity score matching study comparing TARE vs. sorafenib. All studies were of intermediate to low methodological quality. After excluding papers not presenting the outcomes of interest (OS or TTP) in a suitable form for the meta-analysis, a pooled summary estimate on the 21 remaining studies confirmed the good efficacy performance of TARE in HCC unsuitable for curative treatment options.

Included studies suggest that median OS in patients receiving TARE for intermediate-advanced HCC falls in the range of 12-24 months, halved to 6-12 months should PVT be present. Pooled survival rates at 3-years after TARE were estimated as $13 \%$ (with PVT) and 27\% (without PVT). These results are consistent with those presented in other published studies reporting median OS of 7-41.6 months in BCLC B to C patients [41].

Residual heterogeneity among studies - as measured by $\mathrm{I}^{2}$ - was observed particularly in TTP while being less evident for OS. This reflected different schedules and protocols for definition and identification of tumour progression, which is considered a secondary endpoint in the large majority of studies [42]. High heterogeneity was detected also when definition of liver decompensation related to TARE was taken into account; this forced us to compute and summarize data on adverse events only in a qualitative fashion. Despite such limitation, TARErelated liver toxicity was registered in less than $30 \%$ of patients with near to zero mortality directly related to the procedure and an average hospital stay claimed as shorter or equal compared to conventional chemoembolization [13].

This review has a number of limitations. First, only English language studies were included: considering that several clinical studies retrieved were conducted in non-English speaking countries, it is possible that some evidence on the clinical role of TARE has been overlooked. Moreover, individual level data used in the selected studies were not accessible. Individual rather than aggregate data, would have enriched the overall analysis [43].

As regards to the clinical outcomes, the number of papers in which post-progression survival after TARE is described is very limited, although sorafenib is used quite often as second-line therapy after TARE, thus affecting post-progression and overall survival. This was an unavoidable bias assumed to be equally distributed among the studies and therefore not considered as an exclusion driver. Inclusion of post-progression therapies reflected in fact the field practice adopted in the management of HCC patients.

The evidence that supports the use of radioembolization in $\mathrm{HCC}$ is based on retrospective or prospective cohort studies and no RCTs have been published comparing TARE with systemic therapies, in particular sorafenib, currently the mainstay for treating advanced HCC.

Large-scale randomized controlled trials with overall survival as a primary endpoint exploring this comparison have been started (SIRveNIB NCT01135056, SARAH NCT01482442, YES-P NCT01887717). These 


\section{Study}

6 Months

Mazzaferro (2013)

She (2014)

Ozkan (2015)

Khor (2014)

Moreno-Luna (2013)

Hilgard (2010)

El Fouly (2015)

Kolligs (2015)

Kwok (2014)

Woodal (2009)

Subtotal $\left(I^{\wedge} 2=64.07 \%, p=0.00\right)$

\section{Months}

She (2014)

Mazzaferro (2013)

Khor (2014)

Hilgard (2010)

Moreno-Luna (2013)

El Fouly (2015)

Kwok (2014)

Woodal (2009)

Ozkan (2015)

Kolligs (2015)

Subtotal $\left(I^{\wedge} 2=36.55 \%, p=0.12\right)$

\section{Months}

She (2014)

Woodal (2009)

El Fouly (2015)

Kwok (2014)

Khor (2014)

Moreno-Luna (2013)

Mazzaferro (2013)

Ozkan (2015)

Hilgard (2010)

Subtotal $\left(\left.\right|^{\wedge} 2=0.00 \%, p=0.45\right)$

\section{Months}

El Fouly (2015)

Kwok (2014)

Khor (2014)

Mazzaferro (2013)

Moreno-Luna (2013)

Woodal (2009)

Subtotal $\left(\left.\right|^{\wedge} 2=0.00 \%, p=0.85\right)$

\section{Months}

She (2014)

Kwok (2014)

Khor (2014)

El Fouly (2015)

Mazzaferro (2013)

Woodal (2009)

Moreno-Luna (2013)

Subtotal $\left(l^{\wedge} 2=0.00 \%, p=0.85\right)$

\section{EP $\quad 95 \% \mathrm{Cl} \quad$ Weight}

$1.00(0.80,1.00) \quad 8.43$

$1.00(0.63,1.00) \quad 5.48$

$0.88(0.64,0.99) \quad 8.43$

$0.82(0.70,0.91) \quad 13.29$

$0.80(0.68,0.89) \quad 13.24$

$0.77(0.66,0.86) \quad 13.83$

$0.73(0.57,0.85) \quad 12.19$

$0.62(0.32,0.86) \quad 7.33$

$0.61 \quad(0.36,0.83) \quad 8.67$

$0.55(0.32,0.77) \quad 9.11$

$0.80(0.71,0.87) \quad 100.00$

$1.00(0.63,1.00) \quad 3.89$

$0.71 \quad(0.44,0.90) \quad 7.03$

$0.69 \quad(0.56,0.80) \quad 15.58$

$0.67 \quad(0.55,0.77) \quad 16.96$

$0.59(0.46,0.71) \quad 15.45$

$0.57 \quad(0.41,0.72) \quad 13.08$

$0.56(0.31,0.78) \quad 7.33$

$0.55(0.32,0.77) \quad 7.91$

$0.53(0.28,0.77) \quad 7.03$

$0.46(0.19,0.75) \quad 5.74$

$0.63(0.56,0.70) \quad 100.00$

$0.50(0.16,0.84) \quad 2.60$

$0.40(0.19,0.64) \quad 6.28$

$0.39 \quad(0.24,0.55) \quad 13.63$

$0.33(0.13,0.59) \quad 5.67$

$0.31 \quad(0.20,0.44) \quad 19.14$

$0.30 \quad(0.19,0.43) \quad 18.84$

$0.29(0.10,0.56) \quad 5.36$

$0.29(0.10,0.56) \quad 5.36$

$0.20(0.12,0.31) \quad 23.12$

$0.29(0.24,0.35) \quad 100.00$

$0.36(0.22,0.52) \quad 19.78$

$0.33(0.13,0.59) \quad 8.22$

$0.31 \quad(0.20,0.44) \quad 27.78$

$0.29(0.10,0.56) \quad 7.78$

$0.25(0.14,0.37) \quad 27.33$

$0.25(0.09,0.49) \quad 9.11$

$0.29(0.23,0.36) \quad 100.00$

$0.38(0.09,0.76)$

$0.33(0.13,0.59)$

$0.31(0.20,0.44)$

$0.30(0.17,0.45)$

$0.29(0.10,0.56)$

$0.25(0.09,0.49)$

$0.20(0.11,0.32)$

$0.27(0.21,0.33)$
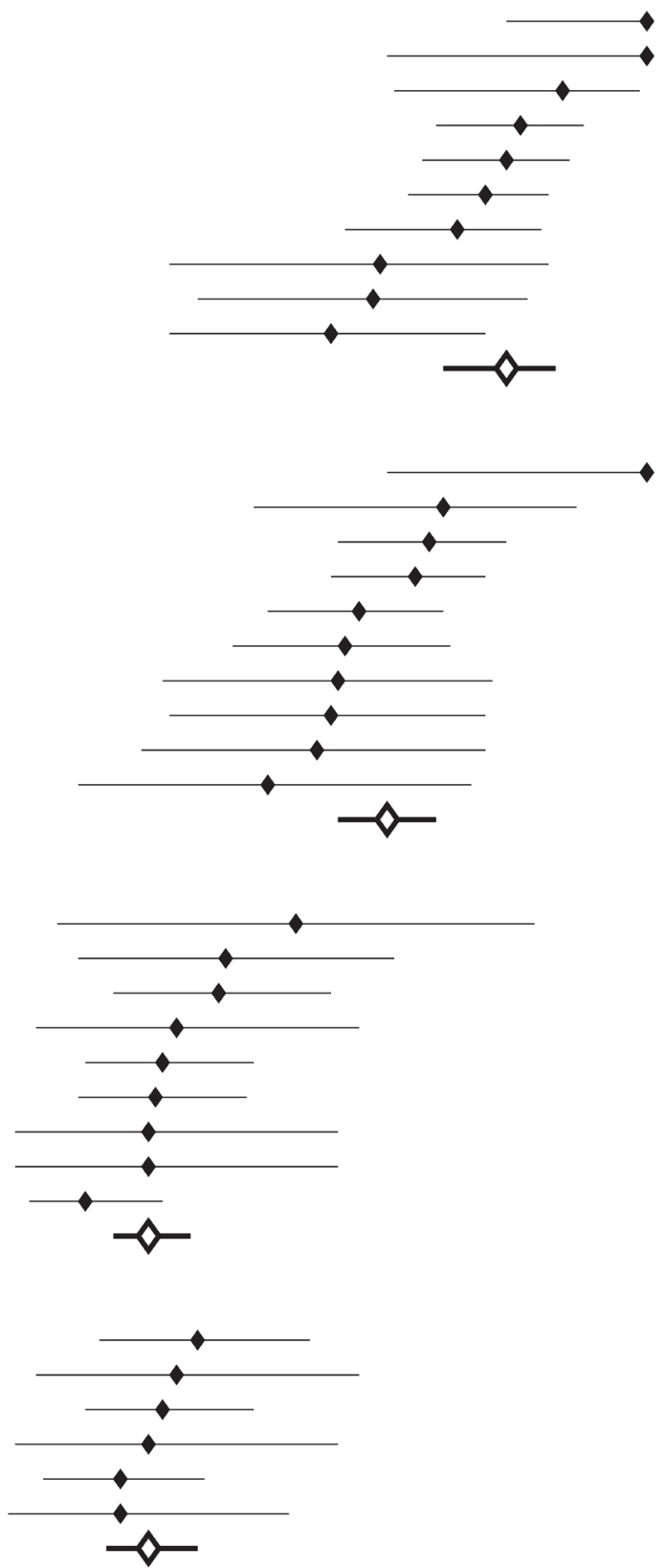

3.64

7.92

26.77

19.06

7.49

8.78

26.34

100.00

\section{A}

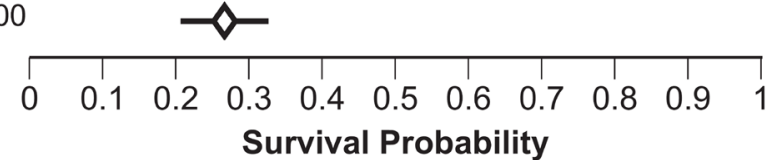




\section{Study}

6 Months

Ozkan (2015)

Mazzaferro (2013)

Hilgard (2010)

Weng (2013)

Khor (2014)

She (2014)

Tsai (2010)

Kwok (2014)

Woodal (2009)

Subtotal $\left(I^{\wedge} 2=74.33 \%, p=0.00\right)$

\section{Months}

Ozkan (2015)

She (2014)

Mazzaferro (2013)

Weng (2013)

Hilgard (2010)

Tsai (2010)

Khor (2014)

Kwok (2014)

Woodal (2009)

Subtotal $\left(I^{\wedge} 2=72.55 \%, p=0.00\right)$

\section{Months}

Hilgard (2010)

Weng (2013)

Ozkan (2015)

Mazzaferro (2013)

Tsai (2010)

She (2014)

Kwok (2014)

Khor (2014)

Subtotal $\left(I^{\wedge} 2=60.45 \%, p=0.01\right)$

\section{Months}

Ozkan (2015)
Tsai $(2010)$
Weng $(2013)$
She $(2014)$
Mazzaferro (2013)
Kwok $(2014)$
Khor $(2014)$
Subtotal $\left(I^{\wedge} 2=0.00 \%, p=0.39\right)$
36 Months
Ozkan $(2015)$
Weng $(2013)$
Mazzaferro $(2013)$
Kwok $(2014)$
Khor $(2014)$
Subtotal $\left(I^{\wedge} 2=0.00 \%, p=0.45\right)$
B

ES $\quad 95 \% \mathrm{Cl}$ Weight

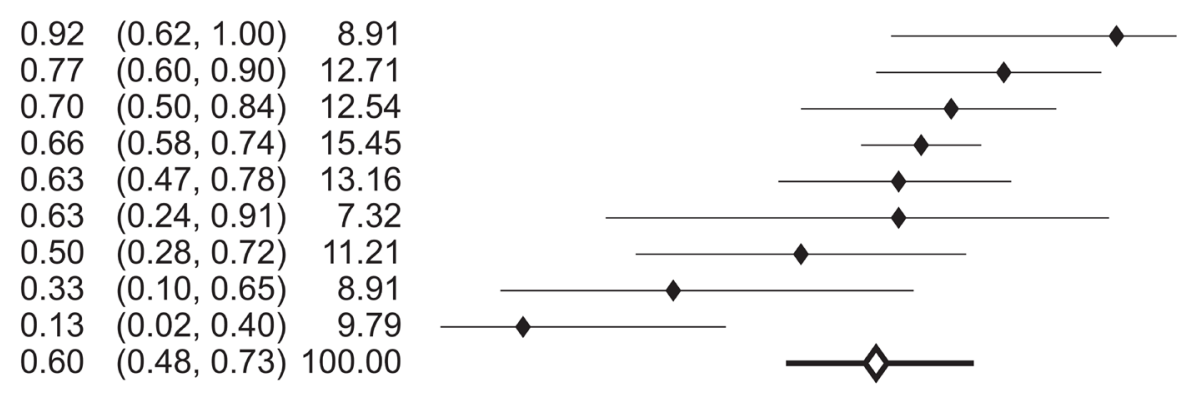

$\begin{array}{lll}0.67 & (0.35,0.90) & 8.78 \\ 0.63 & (0.24,0.91) & 7.14\end{array}$

$0.63 \quad(0.24,0.91) \quad 7.14$

$0.54 \quad(0.37,0.71) \quad 12.79$

$0.46 \quad(0.37,0.54) \quad 15.78$

$0.45 \quad(0.28,0.64) \quad 12.60$

$0.36 \quad(0.17,0.59) \quad 11.18$

$0.24 \quad(0.12,0.40) \quad 13.27$

$0.08 \quad(0.00,0.38) \quad 8.78$

$0.07 \quad(0.00,0.32) \quad 9.68$

$0.37(0.26,0.50) 100.00$

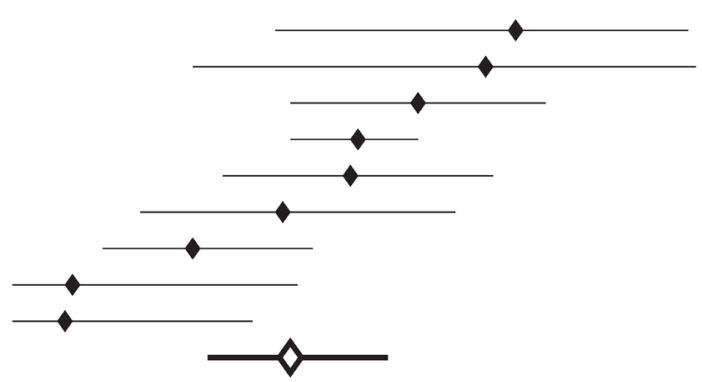

$0.45 \quad(0.28,0.64) \quad 14.18$

$0.26 \quad(0.19,0.34) \quad 20.34$

$\begin{array}{llll}0.25 & (0.05,0.57) \quad 8.57\end{array}$

$0.20 \quad(0.08,0.37) \quad 14.50$

$0.18 \quad(0.05,0.40) \quad 11.91$

$0.13 \quad(0.00,0.53) \quad 6.59$

$0.08 \quad(0.00,0.38) \quad 8.57$

$0.07 \quad(0.02,0.20) \quad 15.34$

$0.12(0.08,0.18) 100.00$
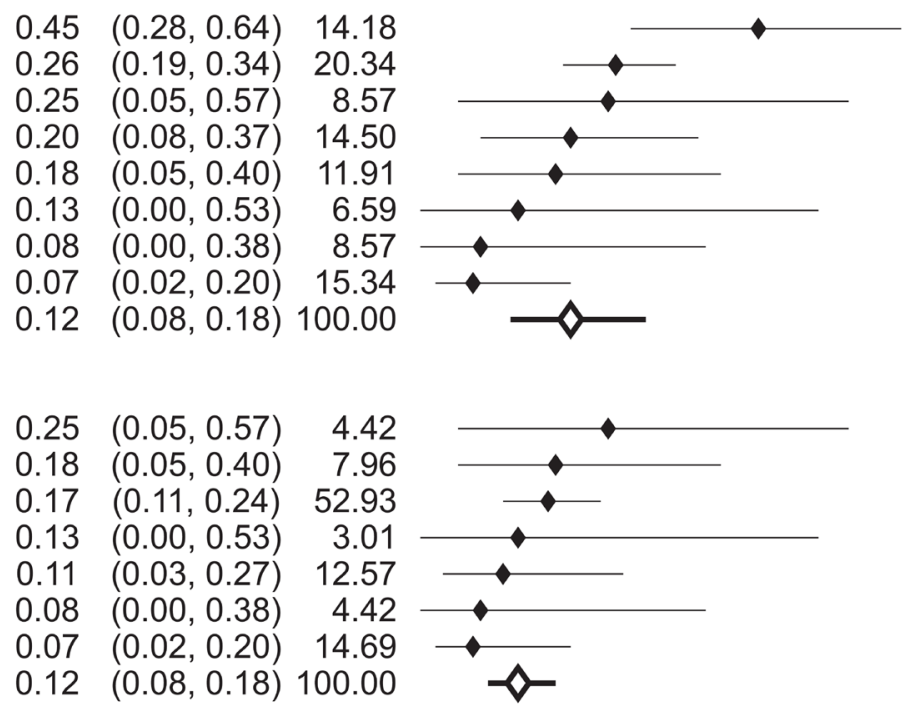

$0.25 \quad(0.05,0.57) \quad 4.97$

$0.17 \quad(0.11,0.24) \quad 59.44$

$0.11 \quad(0.03,0.27) \quad 14.12$

$0.08 \quad(0.00,0.38) \quad 4.97$

$0.07 \quad(0.02,0.20) \quad 16.50$

$0.13(0.09,0.18) 100.00$

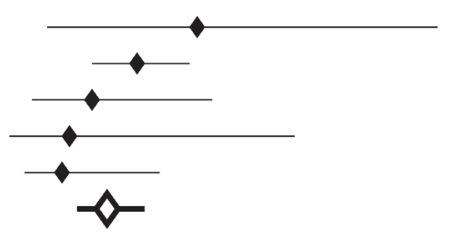

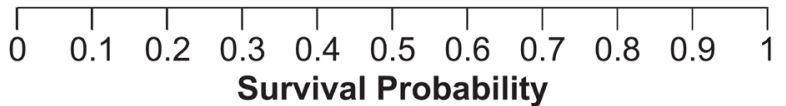

Figure 2: A. Overall survival rates at different follow-up times in intermediate-advanced HCC patients without PVT receiving TARE B. Overall survival rates at different follow-up times in advanced HCC patients with PVT receiving TARE 
trials and future prospective randomized studies of TARE vs. suitable comparators, are crucial to provide direct evidence to evaluate comparative effectiveness because, at least in advanced HCC radioembolization may achieve overall survivals in the range of 6-10 months [27] competitive with the survival range (6.5-10.7 months) reported in the phase III clinical trials registering sorafenib as a standard of care.

Unfortunately, a pooled analysis focused on tumor response was not feasible due to high heterogeneity in reporting this outcome across the included studies. In fact, different response criteria (i.e. Response Evaluation

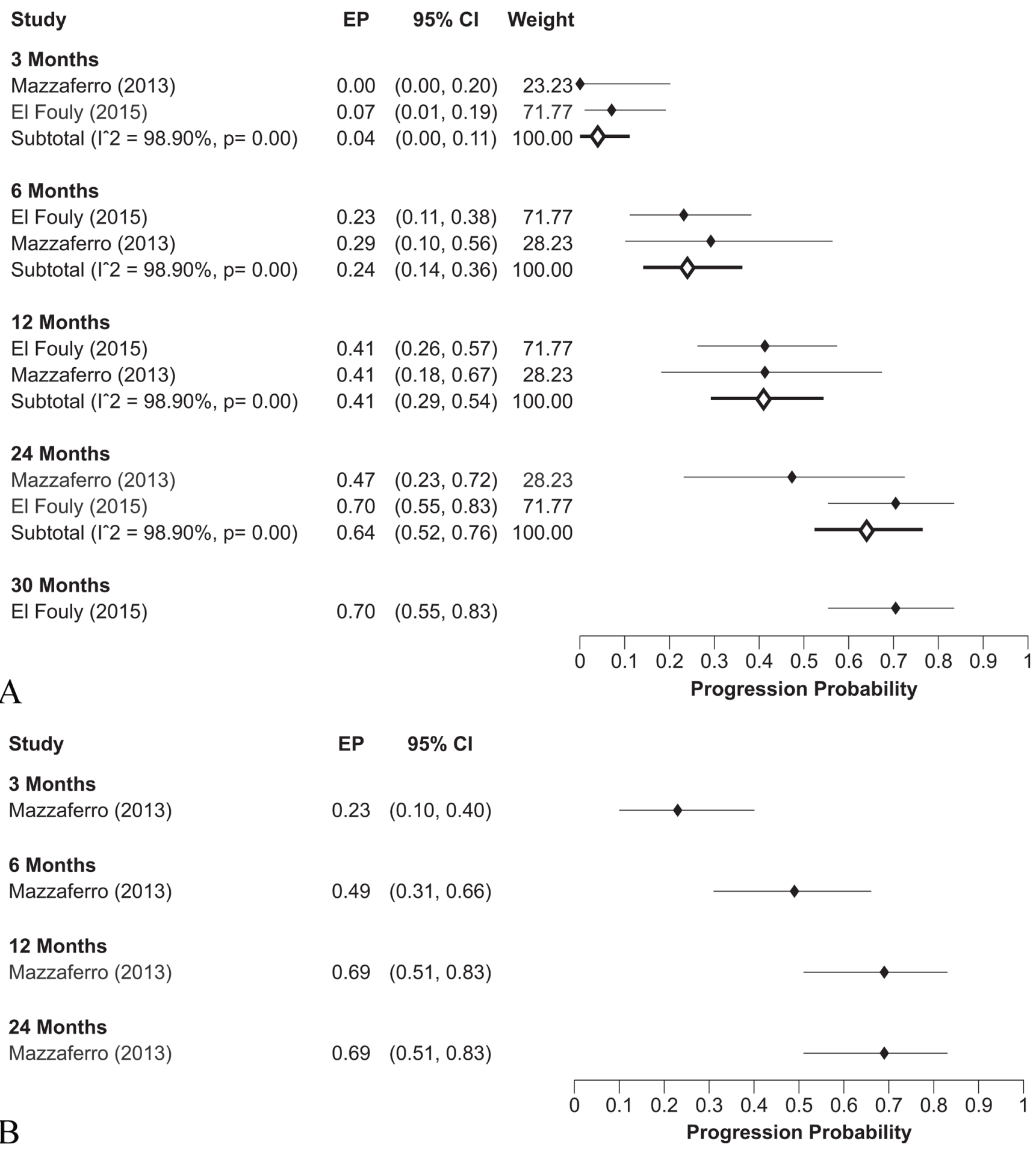

Figure 3: A. Probability of tumor progression at different follow-up times in intermediate-advanced HCC patients without PVT receiving TARE. B. Probability of tumor progression at different follow-up times in advanced HCC patients with PVT receiving TARE. 
Table 3: PICOS inclusion criteria.

\begin{tabular}{|c|c|}
\hline Population & 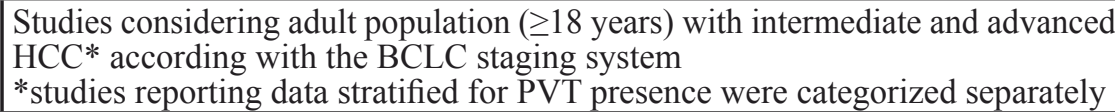 \\
\hline Intervention & TARE using $\mathrm{Y}^{90}$-embedded glass or resin microspheres \\
\hline Comparator & Not specified \\
\hline Outcome measures & $\begin{array}{l}\text { Overall survival; recurrence/progression-free survival, time to progression, } \\
\text { safety }\end{array}$ \\
\hline Study types & $\begin{array}{l}\text { Validation studies; controlled clinical trials; randomized controlled trials; } \\
\text { observational studies (case-report, letters, comments, editorials and non- } \\
\text { systematic review were excluded) }\end{array}$ \\
\hline Availability & English; full text \\
\hline Time and place & Date and place limits were not set \\
\hline
\end{tabular}

$\mathrm{HCC}=$ Hepatocellular carcinoma, $\mathrm{BCLC}=$ Barcelona Clinic Liver Cancer classification, TARE $=$ trans-arterial radioembolization, $\mathrm{PVT}=$ portal vein thrombosis

Criteria In Solid Tumors - RECIST, mRECIST, World Health Organization - WHO criteria), imaging techniques, and time of radiological evaluation prevented a reliable assessment of this secondary endpoint. However, tumor response usually represents only a surrogate endpoint whereas OS, which is universally considered the primary outcome in oncological studies, was consistently assessed in our meta-analysis.

Among the studies comparing TACE versus TARE, two used Drug Eluting Beads TACE (DEB-TACE) [26, $29]$ and five conventional TACE (cTACE) $[15,20,22,35$, 36]. Although subgroup analysis according to the adopted TACE regimen was unfeasible due to the low number of studies, we know from previous cohort studies that DEB-TACE and cTACE lead to similar outcomes and comparable side effects in HCC patients [44, 45].

Observational data allow a preliminary assessment of the incremental costs vs. incremental effectiveness (i.e. cost-effectiveness) of TARE vs. sorafenib and help in this setting to assess the value of presumably similar treatment options in field practice. In a recent cost-effectiveness analysis of TARE vs. TACE, patients with advanced BCLC-C were found to benefit from radioembolization at an increased cost [46] while in patients with BCLC-A disease, who formally lack survival benefit from radioembolization, cost-efficacy could be obtained in some specific subgroups, such as PVT or technical unfeasibility of curative approaches. In the future, a cost-effectiveness analysis could be performed comparing TARE with the other available therapies, particularly sorafenib, to identify whether this procedure is cost-effective or not, and to profile HCC subgroups which could benefit from TARE at a reasonable cost. This analysis could be instrumental in helping policy-decision making, while additional postmarketing evidence is collected.

Given the findings of this systematic review, based on safety and efficacy data from included studies, until the results of the ongoing trials become available, radioembolization appears to be a viable treatment option for patients with intermediate-advanced stage HCC.

\section{MATERIALS AND METHODS}

\section{Literature search}

This review adopts the Preferred Reporting Items for Systematic Reviews and Meta-Analysis (PRISMA) statement [47]. In May 2015, a systematic search was conducted on PubMed, Embase, the Cochrane Library and Web of Science databases to retrieve clinical evidence on TARE for HCC. The search strategy was developed using the PICOS (Patient, Intervention, Comparator, Outcome, Study) framework. Boolean operators "AND" and "OR" were used to combine terms, while "NOT" operator was not included following Cochrane indications. Studies were considered if published in English and related to an adult population ( $\geq 18$ years old). Reference lists of the retrieved articles were screened to find additional studies not identified through the original search.

\section{Selection criteria}

Inclusion criteria are shown in Table 3. Given common indications emerging for $\mathrm{Y}^{90}$, the review was conducted with a focus on patients with intermediate and advanced HCC stages according to BCLC staging system (stages $\mathrm{B}$ and $\mathrm{C}$ ), the latter particularly when presenting with PVT.

\section{Data extraction}

Abstracts and full-text selection was conducted independently by two expert reviewers (CR, SS). In case of debate on study eligibility a third senior reviewer (AF) was involved to reach consensus. Data were extracted using a customized template developed in Microsoft Excel based on the PICOS statement. Information recorded included study features, participants', intervention's and 
comparator's characteristics, safety and efficacy outcomes.

\section{Data analysis}

Data referring to TARE were retrieved from all comparative and non-comparative studies identified. Relevant efficacy outcomes (i.e. overall survival - OS and time to progression - TTP - rates) were summarized and graphically presented through forest plots. When necessary, survival rates were derived from digitalization of Kaplan-Meier survival curves using the software Plot Digitizer 2.6.6C. OS was calculated as the difference between the date of the first treatment and the date of death from any cause, or last observation date in case of censoring. TTP was calculated from the first TARE treatment to the first progression at any site. Main adverse events, such as liver impairment, were tabulated and discussed qualitatively.

Survival rates for OS and TTP from different studies were pooled through a random effect meta-analysis of proportions with exact binomial confidence intervals [48]. A test on the summary effect measure is given, as well as a test for heterogeneity, also quantified using the $\mathrm{I}^{2}$ metric [49]: the higher the values (from $0 \%$ to $100 \%$ ) the larger the heterogeneity across studies. Fixed effect metaanalyses were also performed to check the robustness of obtained estimates to alternative models specification. Results are displayed in forest plots according to followup time and relevant patient subgroups. Analyses were performed using Stata ${ }^{\circledR}$ software (StataCorp, version 14).

\section{Quality assessment}

The methodological quality of included studies was assessed according to a modified version of the National Health Service Centre of Review and Dissemination criteria [50]. Following this guidance, no scoring system was adopted; quality assessments were used for descriptive purposes [51].

\section{ACKNOWLEDGMENTS}

The authors would like to acknowledge Dr. Marco Bongini (GI Surgery and Liver Transplantation Unit, Istituto Nazionale Tumori Fondazione IRCCS, National Cancer Institute, Milan) for assistance in manuscript preparation and Dr. Lucio Fumi for intellectual support to this effort.

\section{CONFLICTS OF INTEREST}

All the authors have no conflicts of interest to declare. SB and VM received lectures and educational fees from Nordion Inc, BTG Plc and Bayer Pharma.
VM is partially supported by AIRC (Italian Association for Cancer Research) and INT-Milan institutional grant (5x1000 grant).

No interferences occurred in carrying out the research project and in writing the manuscript whose content is the sole responsibility of the authors.

\section{GRANT SUPPORT}

The present study was funded by ASBM Srl through an unrestricted grant to CERGAS, Bocconi University, Via Roentgen 1, 20136 Milan, Italy.

\section{REFERENCES}

1. El-Serag HB. Hepatocellular carcinoma. N Engl J Med. 2011 Sep 22;365:1118-27.

2. IARC, Globocan 2012, Last accessed online in June 2016 from http://globocan.iarc.fr/Pages/fact_sheets_cancer.aspx.

3. Forner A, Llovet JM, Bruix J. Hepatocellular carcinoma. Lancet 2012;379:1245-55.

4. European Association For The Study Of The Liver; European Organisation For Research And Treatment Of Cancer. EASL-EORTC clinical practice guidelines: management of hepatocellular carcinoma. J Hepatol. 2012 Apr;56:908-43.

5. Pugh RN, Murray-Lyon IM, Dawson JL, Pietroni MC, Williams R. Transection of the oesophagus for bleeding oesophageal varices. Br J Surg 1973;60:646-649.

6. Bruix J, Sherman M; American Association for the Study of Liver Diseases. Management of hepatocellular carcinoma: an update. Hepatology. 2011 Mar;53:1020-2.

7. Rougier P, Mitrya E, Barbareb JC, Taiebc J. Hepatocellular Carcinoma (HCC): An Update. Sem Oncol 2007 ; 34, Supplement 1: S12-S20.

8. Llovet JM, Ricci S, Mazzaferro V, Hilgard P, Gane E, Blanc JF, de Oliveira AC, Santoro A, Raoul JL, Forner A, Schwartz M, Porta C, Zeuzem S et al. Sorafenib in advanced hepatocellular carcinoma. N Engl J Med 2008;359:378-90.

9. Cheng AL, Kang YK, Chen Z, Tsao CJ, Qin S, Kim JS, Luo R, Feng J, Ye S, Yang TS, Xu J, Sun Y, Liang H et al. Efficacy and safety of sorafenib in patients in the AsiaPacific region with advanced hepatocellular carcinoma: a phase III randomised, double-blind, placebo-controlled trial. Lancet Oncol. 2009 Jan;10:25-34.

10. Iavarone M, Cabibbo G, Piscaglia F, Zavaglia C, Grieco A, Villa E, Cammà C, Colombo M; SOFIA (SOraFenib Italian Assessment) study group. Field-practice study of sorafenib therapy for hepatocellular carcinoma: a prospective multicenter study in Italy. Hepatology 2011;54:2055-63.

11. Salem R, Mazzaferro V, Sangro B. Yttrium 90 radioembolization for the treatment of hepatocellular carcinoma: biological lessons, current challenges, and 
clinical perspectives. Hepatology. 2013 Dec;58:2188-97.

12. Verslype C, Rosmorduc O, Rougier P; ESMO Guidelines Working Group. Hepatocellular carcinoma: ESMO-ESDO Clinical Practice Guidelines for diagnosis, treatment and follow-up. Ann Oncol. 2012 Oct;23 Suppl 7:vii41-8.

13. Sangro B, Iñarrairaegui M, Bilbao JI. Radioembolization for hepatocellular carcinoma. J Hepatol. 2012 Feb;56:464-73.

14. Kulik L, Vouche M, Koppe S, Lewandowski RJ, Mulcahy MF, Ganger D, Habib A, Karp J, Al-Saden P, Lacouture M, Cotliar J, Abecassis M, Baker T et al. Prospective Randomized Pilot Study of Y90 +/- Sorafenib as Bridge to Transplantation in Hepatocellular Carcinoma. Journal of Hepatology 2014;61:309-17.

15. Kolligs FT, Bilbao JI, Jakobs T, Iñarrairaegui M, Nagel JM, Rodriguez M, Haug A, D'Avola D, op den Winkel M, Martinez-Cuesta A, Trumm C, Benito A, Tatsch K et al. Pilot randomized trial of selective internal radiation therapy vs. chemoembolization in unresectable hepatocellular carcinoma. Liver Int. 2015;35:1715-21.

16. Gramenzi A, Golfieri R, Mosconi C, Cappelli A, Granito A, Cucchetti A, Marinelli S, Pettinato C, Erroi V, Fiumana S, Bolondi L, Bernardi M, Trevisani F; BLOG (Bologna Liver Oncology Group). Yttrium-90 radioembolization vs sorafenib for intermediate-locally advanced hepatocellular carcinoma: a cohort study with propensity score analysis. Liver Int. 2015 Mar;35:1036-47.

17. Liu MD, Uaje MB, Al-Ghazi MS, Fields D, Herman J, Kuo JV, Milne N, Nguyen TH, Ramsinghani NS, Tokita KM, Tsai FY, Vajgrt DJ, Imagawa DK. Use of Yttrium-90 TheraSphere for the treatment of unresectable hepatocellular carcinoma. Am Surg. 2004;70:947-53.

18. Reardon KA, McIntosh AF, Shilling AT, Hagspiel KD, Al-Osaimi A, Berg C, Caldwell SH, Northup PG, Angle F, Mulder R, Rich TA. Treatment of primary liver tumors with Yttrium-90 microspheres (TheraSphere) in high risk patients: analysis of survival and toxicities. Technol Cancer Res Treat. 2009 Feb;8:71-7.

19. Woodall CE, Scoggins CR, Ellis SF, Tatum CM, Hahl MJ, Ravindra KV, McMasters KM, Martin RC 2nd. Is selective internal radioembolization safe and effective for patients with inoperable hepatocellular carcinoma and venous thrombosis? J Am Coll Surg. 2009;208:375-82.

20. Carr BI, Kondragunta V, Buch SC, Branch RA. Therapeutic equivalence in survival for hepatic arterial chemoembolization and yttrium 90 microsphere treatments in unresectable hepatocellular carcinoma: a two-cohort study. Cancer. 2010;116:1305-14.

21. Hilgard P, Hamami M, Fouly AE, Scherag A, Müller S, Ertle J, Heusner T, Cicinnati VR, Paul A, Bockisch A, Gerken G, Antoch G. Radioembolization with yttrium-90 glass microspheres in hepatocellular carcinoma: European experience on safety and long-term survival. Hepatology 2010;52:1741-9.

22. Kooby DA, Egnatashvili V, Srinivasan S, Chamsuddin A,
Delman KA, Kauh J, Staley CA 3rd, Kim HS. Comparison of yttrium-90 radioembolization and transcatheter arterial chemoembolization for the treatment of unresectable hepatocellular carcinoma. J Vasc Interv Radiol. 2010;21:224-30.

23. Salem R, Lewandowski RJ, Mulcahy MF, Riaz A, Ryu RK, Ibrahim S, Atassi B, Baker T, Gates V, Miller FH, Sato KT, Wang E, Gupta R et al. Radioembolization for hepatocellular carcinoma using Yttrium-90 microspheres: a comprehensive report of long-term outcomes. Gastroenterology 2010;138:52-64.

24. Tsai AL, Burke CT, Kennedy AS, Moore DT, Mauro MA, Dixon RD, Stavas JM, Bernard SA, Khandani AH, O'Neil BH. Use of yttrium-90 microspheres in patients with advanced hepatocellular carcinoma and portal vein thrombosis. J Vasc Interv Radiol. 2010;21:1377-84.

25. Lambert B, Sturm E, Mertens J, Oltenfreiter R, Smeets P, Troisi R, Van Vlierberghe H, Defreyne L. Intraarterial treatment with Y microspheres for hepatocellular carcinoma: 4 years experience at the Ghent University Hospital. Eur J Nucl Med Mol Imaging. 2011;38:2117-2.

26. Lance C, McLennan G, Obuchowski N, Cheah G, Levitin A, Sands M, Spain J, Srinivas S, Shrikanthan S, Aucejo FN, Kim R, Menon KV. Comparative analysis of the safety and efficacy of transcatheter arterial chemoembolization and yttrium-90 radioembolization in patients with unresectable hepatocellular carcinoma. J Vasc Interv Radiol. 2011;22:1697-705.

27. Sangro B, Carpanese L, Cianni R, Golfieri R, Gasparini D, Ezziddin S, Paprottka PM, Fiore F, Van Buskirk M, Bilbao JI, Ettorre GM, Salvatori R, Giampalma E et al. Survival after yttrium-90 resin microsphere radioembolization of hepatocellular carcinoma across Barcelona clinic liver cancer stages: a European evaluation. Hepatology 2011;54:868-78.

28. Mazzaferro V, Sposito C, Bhoori S, Romito R, Chiesa C, Morosi C, Maccauro M, Marchianò A, Bongini M, Lanocita R, Civelli E, Bombardieri E, Camerini T et al. Yttrium-90 radioembolization for intermediate-advanced hepatocellular carcinoma: a phase 2 study. Hepatology 2013;57:1826-37.

29. Moreno-Luna LE, Yang JD, Sanchez W, Paz-Fumagalli R, Harnois DM, Mettler TA, Gansen DN, de Groen PC, Lazaridis KN, Narayanan Menon KV, Larusso NF, Alberts SR, Gores GJ et al. Efficacy and safety of transarterial radioembolization versus chemoembolization in patients with hepatocellular carcinoma. Cardiovasc.Intervent.Radiol 2013;36:714-23.

30. Weng Z, Ertle J, Zheng S, Lauenstein T, Mueller S, Bockisch A, Gerken G, Yang D, Schlaak JF. A new model to estimate prognosis in patients with hepatocellular carcinoma after Yttrium-90 radioembolization. PLoS One. 2013;8:e82225.

31. Khor AY, Toh Y, Allen JC, Ng DC, Kao YH, Zhu G, Choo SP, Lo RH, Tay KH, Teo JY, Goh BK, Burgmans MC, Irani $\mathrm{FG}$ et al. Survival and pattern of tumor progression with 
yttrium-90 microsphere radioembolization in predominantly hepatitis B Asian patients with hepatocellular carcinoma. Hepatol Int. 2014;8:395-404.

32. Kwok PC, Leung KC, Cheung MT, Lam TW, Szeto LT, Chou SQ, Chia NH, Tong CM, Yuen PK, Cheung CH, Law CK. Survival benefit of radioembolization for inoperable hepatocellular carcinoma using yttrium-90 microspheres. J Gastroenterol Hepatol. 2014;29:1897-904.

33. Padia SA, Kwan SW, Roudsari B, Monsky WL, Coveler A, Harris WP. Superselective yttrium-90 radioembolization for hepatocellular carcinoma yields high response rates with minimal toxicity. J Vasc Interv Radiol. 2014;25:1067-73.

34. Saxena A, Meteling B, Kapoor J, Golani S, Danta M, Morris DL, Bester L. Yttrium-90 radioembolization is a safe and effective treatment for unresectable hepatocellular carcinoma: a single centre experience of 45 consecutive patients. Int J Surg. 2014;12:1403-8.

35. She WH, Cheung TT, Yau TC, Chan AC, Chok KS, Chu FS, Liu RK, Poon RT, Chan SC, Fan ST, Lo CM. Survival analysis of transarterial radioembolization with yttrium-90 for hepatocellular carcinoma patients with HBV infection. Hepatobiliary Surg Nutr 2014;3:185-193.

36. El Fouly A, Ertle J, El Dorry A, Shaker MK, Dechêne A, Abdella H, Mueller S, Barakat E, Lauenstein T, Bockisch A, Gerken G, Schlaak JF. In intermediate stage hepatocellular carcinoma: radioembolization with yttrium 90 or chemoembolization? Liver Int. 2015 Feb;35:627-35.

37. Garin E, Rolland Y, Edeline J, Icard N, Lenoir L, Laffont S, Mesbah H, Breton M, Sulpice L, Boudjema K, Rohou T, Raoul JL, Clement B et al. Personalized dosimetry with intensification using 90Y-loaded glass microsphere radioembolization induces prolonged overall survival in hepatocellular carcinoma patients with portal vein thrombosis. J Nucl Med. 2015;56:339-46.

38. Ozkan ZG, Poyanli A, Ucar A, Kuyumcu S, Akyuz F, Keskin S, Saglam S, Yilmaz E, Karaca C, Turkmen C. Favorable survival time provided with radioembolization in hepatocellular carcinoma patients with and without portal vein thrombosis. Cancer Biother Radiopharm. 2015;30:1328.

39. Soydal C, Keskin O, Kucuk ON, Ozkan E, Bilgic S, Idilman R, Kir MK. Prognostic factors for prediction of survival of hepatocellular cancer patients after selective internal radiation therapy. Ann Nucl Med. 2015;29:426-30.

40. Chiesa C, Mira M, Maccauro M, Romito R, Spreafico C, Sposito C, Bhoori S, Morosi C, Pellizzari S, Negri A, Civelli E, Lanocita R, Camerini $\mathrm{T}$ et al. A dosimetric treatment planning strategy in radioembolization of hepatocarcinoma with $90 \mathrm{Y}$ glass microspheres. Q J Nucl Med Mol Imaging. 2012 Dec;56:503-8.

41. Sangro B, Salem R, Kennedy A, Coldwell D, Wasan H. Radioembolization for hepatocellular carcinoma: a review of the evidence and treatment recommendations. Am J Clin Oncol. 2011;34:422-431.
42. Ciani O, Davis S, Tappenden P, Garside R, Stein K, Cantrell A, Saad ED, Buyse M, Taylor RS. Validation of surrogate endpoints in advanced solid tumors: systematic review of statistical methods, results, and implications for policy makers. Int J Technol Assess Health Care. 2014 Jul;30:312-24.

43. Riley RD, Lambert PC, Abo-Zaid G. Meta-analysis of individual participant data: rationale, conduct, and reporting. BMJ. 2010;340:c221.

44. Facciorusso A, Mariani L, Sposito C, Spreafico C, Bongini M, Morosi C, Cascella T, Marchianò A, Camerini T, Bhoori S, Brunero F, Barone M, Mazzaferro V. Drug-eluting beads versus conventional chemoembolization for the treatment of unresectable hepatocellular carcinoma. J Gastroenterol Hepatol. 2016 Mar;31:645-53.

45. Golfieri R, Giampalma E, Renzulli M, Cioni R, Bargellini I, Bartolozzi C, Breatta AD, Gandini G, Nani R, Gasparini D, Cucchetti A, Bolondi L, Trevisani F; PRECISION ITALIA STUDY GROUP. Randomised controlled trial of doxorubicin-eluting beads vs conventional chemoembolisation for hepatocellular carcinoma. Br J Cancer. 2014 Jul 15;111:255-64.

46. Rostambeigi N, Dekarske AS, Austin EE, Golzarian J, Cressman EN. Cost effectiveness of radioembolization compared with conventional transarterial chemoembolization for treatment of hepatocellular carcinoma. J Vasc Interv Radiol. 2014 Jul;25:1075-84.

47. Moher D, Liberati A, Tetzlaff J, Altman DG; PRISMA Group. Preferred reporting items for systematic reviews and meta-analyses: the PRISMA statement. Int J Surg 2010;8:336-41.

48. Newcombe RG. Two-sided confidence intervals for the single proportion: comparison of seven methods. Statistics in Medicine 1998;17: 857-872.

49. Higgins JP, Thompson SG, Deeks JJ, Altman DG. Measuring inconsistency in meta-analyses. BMJ. 2003;327:557-60.

50. Ciani O, Hoyle M, Pavey T, Cooper C, Garside R, Rudin $\mathrm{C}$, Taylor R. Complete cytogenetic response and major molecular response as surrogate outcomes for overall survival in first-line treatment of chronic myelogenous leukemia. A case study for technology appraisal on the basis of surrogate outcomes evidence. Value Health. 2013;16:1081-90.

51. CRD, Systematic Reviews: CRD's guidance for undertaking reviews in health care, 2009: York, University of York CRD. 